\title{
Numerical assessment of the combination of subgridding and the perfectly matched layer grid termination in the finite difference time domain method
}

\author{
Thomas Cuyckens ${ }^{1 *}$, Hendrik Rogier ${ }^{1}$ and Daniel De Zutter ${ }^{1}$ \\ ${ }^{1}$ INTEC, Ghent University, Sint-Pietersnieuwstraat 41, 9000 Gent, Belgium
}

\begin{abstract}
SUMMARY
The Finite Difference Time Domain (FDTD) method is one of the well-established numerical techniques to model electromagnetic problems. In order to be able to efficiently include the finer geometrical details, subgridding is used to avoid the use of a fine mesh over the whole region of interest. Our work builds on and extends the systematic framework provided by Chilton in his $\mathrm{PhD}(\mathrm{H}-\mathrm{P}$ - and T-refinement strategies for the FDTD-method developed via Finite Element principles, Ohio State University, 2008).

A crucial element of the FDTD-method is the termination of the grid in an absorbing boundary condition $(\mathrm{ABC})$. With the invention of the Perfectly Matched Layer (PML) by Bérenger, this PML is now the standard $\mathrm{ABC}$ allowing very low reflection, even when positioned close to scatterers.

In this contribution, the PML is first included into the before mentioned subgridding framework. Next, the interaction between the subgridded problem space and the (subgridded) PMLs is studied. Therefore, different (geometrical) subgridding strategies for the PML are considered. We numerically investigate the effect of these strategies by determining the reflection of a point source caused by the PML. It becomes possible to not only determine the general reflection level, but also to pinpoint the precise source of the reflections. The conclusion of this study is that important reflections are found originating from the corner points of the PML (as expected) but these reflections worsen when grid non-uniformities are present due to subgridding. Hence, the combination of subgridding and PML should be handled with great care.
\end{abstract}

KEY WORDS: Finite Difference Time Domain; subgridding; Perfectly Matched Layer

\section{INTRODUCTION}

Together with Finite Elements and integral equation techniques solved by the Method of Moments, the Finite-Difference Time-Domain (FDTD, [?]) is a well-established numerical method for the fullwave simulation of electromagnetic problems. Amongst others, its advantages over its competitors are: the vastly larger number of unknowns it can solve for, the possibilities to include non-linearities and its suitability to be massively parallelised. On the downside, however, in its basic form, the mesh has to be kept strictly uniform in order to maintain an explicit formulation. Therefore, the inclusion of electrically small design components in a large computational domain leads to either huge memory requirements and unacceptable simulation times, or inaccurate results.

To overcome this problem, many different schemes for (spatial) subgridding of the FDTD mesh were proposed. From those, only a few were passive, stable and reciprocal. And from those, none offered the freedom to choose different refinement factors along different mesh directions. Yet, flexible subgridding would not only provide a far better possibility to design certain structures in FDTD,

\footnotetext{
*Correspondence to: INTEC, Ghent University, Sint-Pietersnieuwstraat 41, 9000 Gent, Belgium. E-mail: thomas.cuyckens@ugent.be 
it also reduces calculation time by not forcing the designer to use the finest cell size everywhere within the simulation domain. This leads to more precise simulation results obtained in a more efficient way.

For open structures, such as, for example, antennas, FDTD implementations require a form of mesh termination that mimics free space. The perfectly matched layer (PML) introduced by JeanPierre Bérenger in 1994 [?] has become the de facto standard for absorbing boundary conditions in FDTD. Although the PML is reflectionless in theory, in practice, after discretization, reflections are introduced in the calculation domain. To minimize these errors, a PML discretization that matches the mesh topology everywhere would be preferable. Up to now, the most optimal combination in terms of memory requirements and computation time of suitable subgridding schemes combined with adequate PML termination strategies is still an important open research issue.

When embedding a finer mesh within a coarser one, special care has to be taken to adapt the update equations for the fields at the interface of the two meshes. To address this problem, various techniques were developed, such as equivalent circuits of grid discontinuities [?], digital filtering [?], recessed interfaces [?], and Huygens subgridding [?,?,?]. These techniques have drawbacks such as, allowing only odd refinement factors, the need of overlapping meshes or transition regions, requiring the inversion of matrices, as well as late time instability issues or non-symmetrical update equations. Next to those problems, most schemes do not provide a lot of flexibility in terms of refinements nor mesh forms. This often results in mesh specific algorithms and complex update equations.

The work of Monk [?] introduced a scheme with several interesting features: meshes of different resolution only share their boundaries, discrete reciprocity and proven stability. A main restriction is that it only allows a 1:2 refinement. In [?], a finite element approach is used to achieve subgridding, laying out an interesting mathematical framework for subgridding, applicable to a whole range of configurations. Chilton combined both concepts to one subgridding algorithm [?] with refinement factors 1: $N$ and later $M: N\left(M, N \in \mathbb{N}_{0}\right)$. This approach was extended to BOR-FDTD in [?].

PMLs have been expressed in all kinds of ways. Originally, they were formulated in terms of splitfield equations [?]. Later, unsplit formulations were put forward, such as the Maxwellian or uniaxial PML (UPML, [?]) and the non-Maxwellian or complex frequency shifted PML (CFS-PML [?]). The PML equations have also been expressed in different coordinate systems [?] and even in a coordinate independent way [?]. This last formulation made use of differential forms [?], exactly the foundation of the subgridding algorithm used here.

Other methods, such as the 'Multiresolution Finite Difference' [?] and 'Pseudo Spectral Time Domain' [?] yield longer simulation times and have boundary implementation problems, respectively, and are therefore less suited in the pursuit of a general fast and efficient simulation tool.

In this paper, we present a generalized FDTD subgridding technique for an open simulation domain with the following new features:

- The limited refinement freedom of [?] is extended to allow (almost) arbitrary mesh refinements that are mesh direction independent and this without losing any of the desirable properties such as reciprocity, passivity and stability.

- As (almost) arbitrary mesh refinements are possible, the influences of normal and tangential (both will be defined later on) subgridding can be studied independently of one another.

- To reduce reflections, avoid undesirable side effects such as standing waves and maximize design freedom, mesh refinements are extended towards PMLs. The subgridding possibility will also lead to some new insights into PML implementations.

In a first part, Maxwell's equations in differential geometry form and the corresponding vector representation are briefly repeated. Next, the introduced concepts are expanded to allow subgridding. In a last part, these concepts will be broadened again to include subgridding of a PML. 


\section{GENERALIZED SUBGRIDDED FDTD FOR OPEN REGIONS}

\subsection{Discrete representation of Maxwell's equations}

In absence of sources, the homogeneous Maxwell equations together with the constitutive laws (with $\tau=c t$ and $\Delta \tau=c \Delta t$ ) are formulated in differential form as

$$
\begin{array}{rlrl}
-\partial_{\tau} B & =\mathrm{d} E & \partial_{\tau} D & =\mathrm{d} H \\
\mathrm{~d} B & =0 & \mathrm{~d} D & =0 \\
B & =\star_{\mu_{r}} H & D & =\star_{\varepsilon_{r}} E
\end{array}
$$

where $\mathrm{d}$ is the exterior derivative operating on a $k$-form. $\star_{\varepsilon_{r}}$ and $\star_{\mu_{r}}$ denote the by the permittivity $\varepsilon_{r}$ and permeability $\mu_{r}$ scaled version of the hodge-star operator, respectively. $E$ and $H$ are 1-forms whereas $B$ and $D$ are 2 -forms. The discretization of the exterior derivative d operating on 1-forms in (1a) (corresponding to the curl operator in classical vector notation) results in the curl stencil matrix $C_{1}$, which represents the signed incidence vector of the faces in the calculation domain $\Omega$. Therefore, 1a can be rewritten in semi-discrete form as

$$
\begin{aligned}
-\partial_{\tau} b & =C_{1} e \\
\partial_{\tau} e & =\left[\star_{\varepsilon_{r}}\right] C_{1}^{T}\left[\star_{\mu_{r}}^{-1}\right] b
\end{aligned}
$$

with the strictly diagonal mass matrices $\left[\star_{\mu_{r}}^{-1}\right]$ and $\left[\star_{\varepsilon_{r}}\right]$ a mass lumped [?] version of

$$
\begin{gathered}
\widetilde{\left[\star_{\mu_{r}}^{-1}\right]}=\int_{\Omega} W_{2} \mu_{r}^{-1} W_{2}^{T} \mathrm{~d} \Omega \\
\widetilde{\left[\star_{\varepsilon_{r}}\right]}=\int_{\Omega} W_{1} \varepsilon_{r} W_{1}^{T} \mathrm{~d} \Omega
\end{gathered}
$$

with $W_{k}$ Whitney-forms [?] of order $k$ used to discretely expand $E$ and $B$ into $e$ and $b$. The explicit fully discrete update equations are obtained by replacing the time derivative by its central difference in its classical staggered way.

\subsection{Arbitrary mesh refinements}

Consider a (coarse) uniform background FDTD mesh consisting of fixed cells with constant dimensions $[\Delta x, \Delta y, \Delta z]$ in the $(x, y, z)$ directions. In order to outline our arbitrary mesh refinement strategy we focus on two locally refined meshes $M^{p}$ and $M^{q}$, characterized by absolute refinement vectors $N^{p}=\left[N_{x}^{p}, N_{y}^{p}, N_{z}^{p}\right]$ and $N^{q}=\left[N_{x}^{q}, N_{y}^{q}, N_{z}^{q}\right]$, respectively. Hence, the cells sizes in meshes $M^{p}$ and $M^{q}$ were reduced to $\left(\Delta x / N_{x}^{p}, \Delta y / N_{y}^{p}, \Delta z / N_{z}^{p}\right)$ and $\left(\Delta x / N_{x}^{q}, \Delta y / N_{y}^{q}, \Delta z / N_{z}^{q}\right)$, respectively. To connect the two meshes at their interface, we introduce a local coordinate system $\left(n, t_{1}, t_{2}\right)$, with $n$ the normal direction along which the meshes are stitched together, and $t_{1}$ and $t_{2}$ two tangential orthogonal unit vectors spanning the plane of the interface. The relative mesh refinement when joining $M^{p}$ and $M^{q}$ at their interface is characterized by the relative mesh refinement vector $\nu$, defined by

$$
\nu=\left[\nu_{n}, \nu_{t_{1}}, \nu_{t_{2}}\right]=\left[\max \left(\frac{N_{n}^{p}}{N_{n}^{q}}, \frac{N_{n}^{q}}{N_{n}^{p}}\right), \max \left(\frac{N_{t_{1}}^{p}}{N_{t_{1}}^{q}}, \frac{N_{t_{1}}^{q}}{N_{t_{1}}^{p}}\right) \max \left(\frac{N_{t_{2}}^{p}}{N_{t_{2}}^{q}}, \frac{N_{t_{2}}^{q}}{N_{t_{2}}^{p}}\right)\right]
$$

where $\max (x, y)$ represents the maximum of the two arguments $x$ and $y$. In addition, we introduce $\vartheta_{i}=\arg \max \left(\frac{N_{i}^{p}}{N_{i}^{q}}, \frac{N_{i}^{q}}{N_{i}^{p}}\right)$, as the index of the largest of the two elements, for $i$ either $n, t_{1}$ or $t_{2}$. Now, we outline the general procedure to stitch together $M^{p}$ and $M^{q}$, provided the following two restrictions apply:

(1) $\nu_{t_{1}}$ and $\nu_{t_{2}} \in \mathbb{N}_{0}$ 


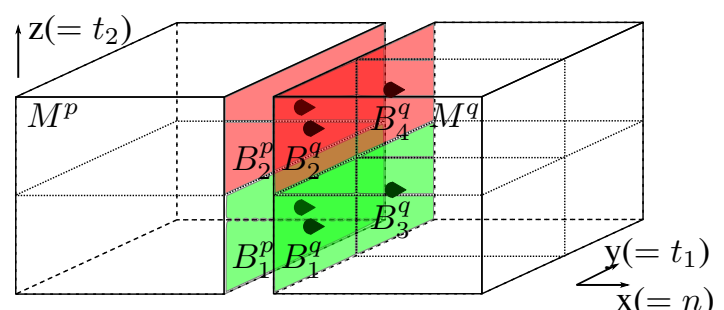

(a) Valid subgridding with factors $N^{p}=[1,1,2]$ and $N^{q}=[1,2,2]$

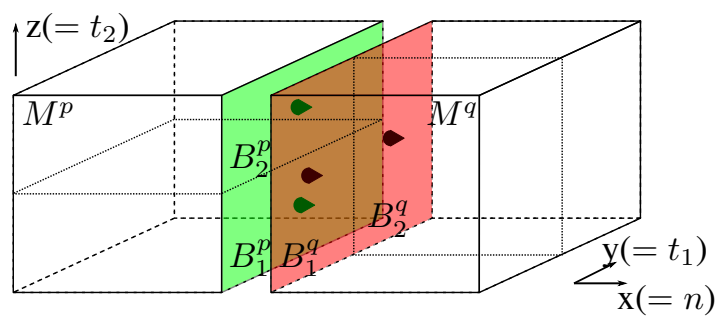

(b) Invalid subgridding with factors $N^{p}=$ $[1,1,2]$ and $N^{q}=[1,2,1]$

Figure 1

(2) $\vartheta_{t_{1}}=\vartheta_{t_{2}}$

The first condition can be relaxed to $\mathbb{Q}_{0}$, as shown in [?]. To better understand the second restriction, consider Fig. 1a, illustrating a valid (a) and an invalid (b) subgridding scheme. On the one hand, for case (a), meshes $M^{p}$, with absolute refinement vector $N^{p}=[1,1,2]$, and $M^{q}$, with absolute refinement vector $N^{q}=[1,2,2]$, may not be joined, as their relative refinement vector is given by $\nu=[1,2,1]$ whereas the index vector is found to be $\vartheta=\left[\vartheta_{n}, \vartheta_{t_{1}}, \vartheta_{t_{2}}\right]=[1$ or $2,2,1$ or 2$]$, such that $\vartheta_{t_{1}}=\vartheta_{t_{2}}=2$. The latter condition in general implies that each normal $B$-field of $M^{p}$ that lies in the subgridding interface has to be a sum of normal $B$-fields of $M^{q}$ in the subgridding interface or vice versa. In case (a), the configuration in Fig. $1 \mathrm{a}$ is valid, because $B_{1}^{p}=B_{1}^{q}+B_{3}^{q}$ and $B_{2}^{p}=B_{2}^{q}+B_{4}^{q}$. On the other hand, for case (b), meshes $M^{p}$, with absolute refinement vector $N^{p}=[1,1,2]$, and $M^{q}$, with absolute refinement vector $N^{q}=[1,2,1]$, may be joined, as their relative refinement vector is given by $\nu=[1,2,2]$ whereas the index vector is found to be $\vartheta=\left[\vartheta_{n}, \vartheta_{t_{1}}, \vartheta_{t_{2}}\right]=[1$ or $2,2,1]$, such that $\vartheta_{t_{1}}=2 \neq \vartheta_{t_{2}}=1$. Hence, the set up of Fig. $1 \mathrm{~b}$ is not allowed since $B_{1}^{p} \neq B_{1}^{q}+B_{2}^{q}$ and $B_{1}^{q} \neq B_{1}^{p}+B_{2}^{p}$.

Consider a coarse mesh $M^{p}$ and a fine mesh $M^{q}$. To join both meshes, tangential edges and normal faces that occur twice at the subgridding interface must be eliminated. In particular, the field/induction components occurring at the interface of the fine mesh will be removed. Associate with the edges corresponding to the normal $n$-, tangential $t_{1}$ - and tangential $t_{2}$-components of the electric field in the meshes $M^{p}$ and $M^{q}$ the expansion coefficient vectors $e_{n}^{p}, e_{t_{1}}^{p}, e_{t_{2}}^{p}$ and $e_{n}^{q}, e_{t_{1}}^{q}, e_{t_{2}}^{q}$, respectively, and with the faces corresponding to the normal $n-$, tangential $t_{1}$ - and tangential $t_{2}$-components of the magnetic induction in the meshes $M^{p}$ and $M^{q}$ the expansion coefficient vectors $b_{n}^{p}, b_{t_{1}}^{p}, b_{t_{2}}^{p}$ and $b_{n}^{q}, b_{t_{1}}^{q}, b_{t_{2}}^{q}$, respectively. The elimination of the fine forms is performed by means of restriction matrices $A_{e}$ and $A_{b}$ (associated with electric field or magnetic induction components, respectively). Each restriction matrix is a diagonal block matrix, composed by restriction matrices acting on one particular component, hence $A_{e}=\operatorname{diag}\left(A_{e, n}, A_{e, t_{1}}, A_{e, t_{2}}\right)$ and $A_{b}=\operatorname{diag}\left(A_{b, n}, A_{b, t_{1}}, A_{b, t_{2}}\right)$. Each restriction matrix composing $A_{e}$ or $A_{b}$ is constructed in an identical fashion. Let $A_{x, y}$ denote one such matrix for component $y\left(n, t_{1}\right.$ or $\left.t_{2}\right)$ of field $x$ ( $e$ or b). Its construction is implemented by means of the following steps:

(i) Let $x_{y, i}^{p}$ denote the $i^{\text {th }}$ expansion coefficient in field/induction component vector $x_{y}^{p}$ and $x_{y, j}^{q}$ the $j^{\text {th }}$ expansion coefficient in field/induction component vector $x_{y}^{q}$.

(ii) Create a block matrix $A_{k}^{\prime}=\operatorname{diag}\left(\mathrm{I}_{\alpha}, \mathrm{I}_{\beta}\right)$ with $\mathrm{I}_{\alpha}$ and $\mathrm{I}_{\beta}$ unit matrices of order $i_{\text {max }}$, equal to the total number of $y$-oriented edges/faces in $M^{p}$, and $j_{\text {max }}$ corresponding to the total number of $y$-oriented edges/faces in $M^{p}$, respectively.

(iii) $A_{k}^{\prime}\left[i, i_{\max }+j_{m}\right]=1, \exists\left\{x_{y, j_{m}}^{q}\right\}: x_{y, i}^{p}=\sum_{m} x_{y, j_{m}}^{q}$

(iv) $A_{k}^{\prime}\left[i_{1}, i_{\text {max }}+j\right]=\gamma, A_{k}^{\prime}\left[i_{2}, i_{\text {max }}+j\right]=1-\gamma, \exists x_{y, i_{1}}^{p}, x_{y, i_{2}}^{p} \in\left\{\operatorname{nb}_{c}\left(x_{y, j}^{p}\right)\right\}$

(v) delete all rows $\left(i_{\max }+j\right)$ that satisfied either (iii) or (iv).

(vi) now $A_{k}=A_{k}^{\prime}$ 


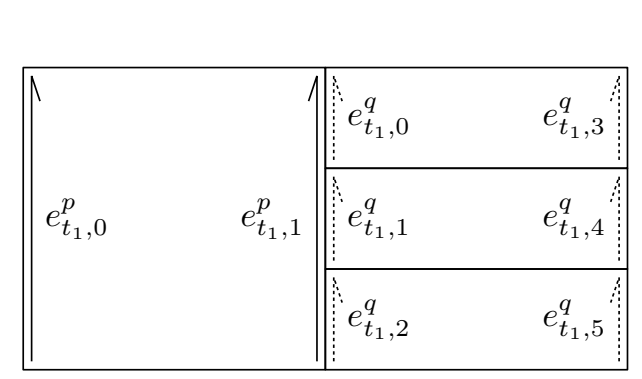

(a) $e_{t_{1}, 1}^{p}=e_{t_{1}, 0}^{q}+e_{t_{1}, 1}^{q}+e_{t_{1}, 2}^{q}\left(\right.$ see $\left.A_{e, t_{1}}\right)$

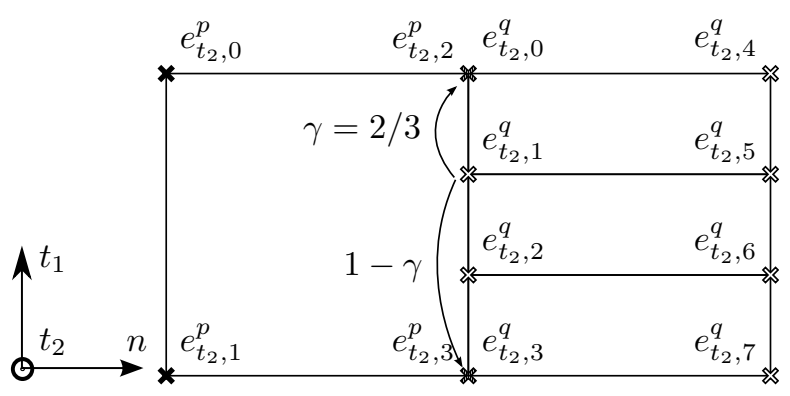

(b) $e_{t_{2}, 2}^{p}, e_{t_{2}, 3}^{p} \in\left\{\operatorname{nb}_{c}\left(e_{t_{2}, 1}^{q}\right)\right\}\left(\right.$ see $\left.A_{e, t_{2}}\right)$

Figure 2. Illustration of step iii)(left) and iv (right) in the construction of $A_{1}$

where $\gamma$ is inversely proportional to the distance to $x_{y, i_{1}}^{p}$ and $\left\{\operatorname{nb}_{c}\left(x_{y, j}^{p}\right)\right\}$ is the set containing the coarse form neighbours of fine form $\beta_{j}^{k}$.

Steps (iii) and (iv) are illustrated by Fig. 2, which focuses on the discretization of the $t_{1}$ tangential electric fields at the interface. The labelling is done from top to bottom and left to right (thus $0 \leq i \leq 1$ and $0 \leq j \leq 5$. The construction of $A_{e, t_{1}}$, given the expansion coefficient vectors $e_{t_{1}}^{p}$ and $e_{t_{1}}^{q}$ in Fig. 2a proceeds as follows:

$$
\begin{aligned}
& A_{e, t_{1}}^{\prime} \stackrel{(i i)}{=}\left[\begin{array}{c:c}
\mathrm{I}_{2} & 0_{2 \times 6} \\
\hdashline 0_{6 \times 2} & \mathrm{I}_{6}
\end{array}\right] \stackrel{\text { iii }}{\Rightarrow}\left[\begin{array}{c:cccc}
\mathrm{I}_{2} & 0 & 0 & 0 & 0_{2 \times 3} \\
\hdashline & 1 & 1 & 1 \\
\hdashline & \mathrm{I}_{3} & 0_{3 \times 3} \\
0_{6 \times 2} & 0_{3 \times 3} & \mathrm{I}_{3}
\end{array}\right] \leftarrow \text { delete } \\
& \stackrel{\text { v. }}{\Rightarrow}\left[\begin{array}{c:cccc}
\mathrm{I}_{2} & 0 & 0 & 0 & 0_{2 \times 3} \\
\hdashline 0_{3 \times 2} & 0_{3 \times 3} & \mathrm{I}_{3}
\end{array}\right] \stackrel{v i i}{=} A_{e, t_{1}}
\end{aligned}
$$

The resulting matrix removes three fine edges, resulting in a mesh with five edges (two coarse $\left(e_{t_{1}, 0}^{p}\right.$ and $\left.e_{t_{1}, 1}^{p}\right)$ and three fine $\left(e_{t_{1}, 3}^{q}\right.$ to $\left.\left.e_{t_{1}, 5}^{q}\right)\right)$, hence $A_{e, t_{1}}$ is a 5 by 8 matrix.

Next, $A_{e, t_{2}}$ is constructed by considering the expansion vectors containing the $t_{2}$-oriented electric fields $e_{t_{2}}^{p}$ and $e_{t_{2}}^{q}$ in Fig. 2b. The labelling is done again in a top-down/left-right fashion (thus $0 \leq i \leq 3$ and $0 \leq j \leq 7$. Therefore

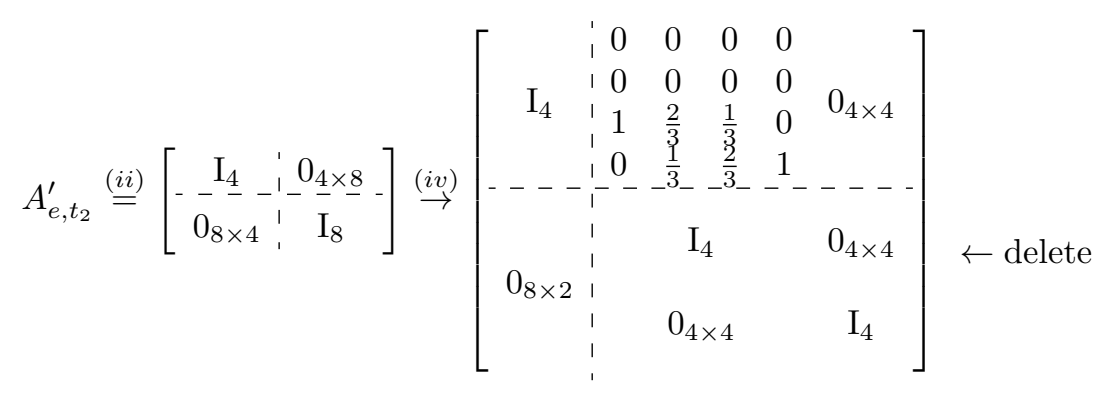




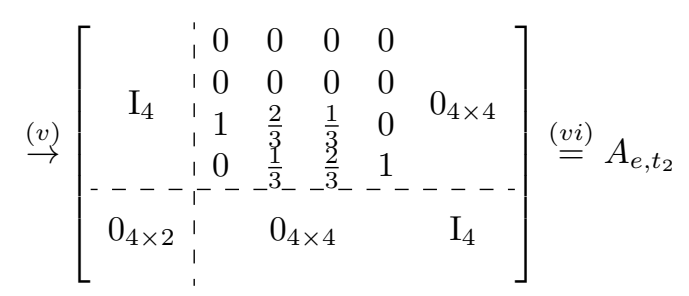

The resulting 8 by 12 matrix illustrates that the restriction operator reduced the number of redundant edges from twelve to eight (four coarse $\left(e_{t_{2}, 0}^{p}\right.$ to $\left.e_{t_{2}, 3}^{p}\right)$ and four fine edges $\left(e_{t_{2}, 4}^{q}\right.$ to $\left.e_{t_{2}, 7}^{q}\right)$ ).

Next construct a block matrix $C_{1}^{p q}$ consisting of the curl stencil matrices $C_{1}^{p}$ and $C_{2}^{p}$ of meshes $M^{p}$ and $M^{q}$, respectively.

$$
C_{1}^{p q}=\left[\begin{array}{cc}
C_{1}^{p} & 0 \\
0 & C_{1}^{q}
\end{array}\right]
$$

(2) may still be used, provided that $C_{1}$ is redefined as

$$
C_{1}=A_{2}^{+} C_{1}^{p q} A_{1}^{T}
$$

which can be proven, using the fact that $A_{1} C_{1}^{p q T}=C_{1}^{T} A_{2}$ and where $A^{+}$is the Moore-Penrose (or pseudo) inverse of $A$. Moreover, as a result of introducing $A_{1}$ and $A_{2}$, (3) changes to

$$
\begin{gathered}
\widetilde{\left[\star_{\mu_{r}}^{-1}\right]}=A_{2}\left(\int_{\Omega} W_{2} \mu_{r}^{-1} W_{2}^{T} \mathrm{~d} \Omega\right) A_{2}^{T} \\
\widetilde{\left[\star_{\varepsilon_{r}}\right]}=A_{1}\left(\int_{\Omega} W_{1} \varepsilon_{r} W_{1}^{T} \mathrm{~d} \Omega\right) A_{1}^{T}
\end{gathered}
$$

\subsection{Perfectly Matched Layer}

In case of a Perfectly Matched Layer (PML), the semi-discrete Maxwell equations (2) are modified as [?]:

$$
\begin{aligned}
-\partial_{\tau} \Lambda_{2} b & =C_{1} e \\
\partial_{\tau} \Lambda_{1} e & =\left[\star_{\varepsilon_{r}}\right] C_{1}^{T}\left[\star_{\mu_{r}}^{-1}\right] b
\end{aligned}
$$

where matrices $\Lambda_{1}$ and $\Lambda_{2}$ were introduced as the result of a complex coordinate transformation of the field variables of the form [?]:

$$
x^{i} \rightarrow \tilde{x}^{i}=\int_{0}^{x^{i}} s_{i}(\eta) \mathrm{d} \eta \quad i \in\{0,1,2\},
$$

with $x^{i}$ the $i$ th component of the base spanning the space. Following [?] $s_{i}$ is defined by

$$
s_{i}=\left\{\begin{array}{cl}
\kappa_{i}+\frac{\sigma_{i}}{j \omega \varepsilon_{0}} & \text { PML absorbs in } i \text { direction } \\
1 & \text { PML does not absorbs in } i \text { direction }
\end{array}\right.
$$

The stretching matrices are then constructed as follows:

$$
\begin{aligned}
& \tilde{\Lambda}_{1}[j, k]=\sum_{\ell} \hat{y}[m] \cdot \phi(\ell) \cdot \hat{y}[r] \int_{\Omega_{\ell}} W_{1}[m] W_{1}^{T}[r] \mathrm{d} \Omega \\
& \tilde{\Lambda}_{2}[j, k]=\sum_{\ell} \hat{y}[m] \cdot \phi(\ell) \cdot \hat{y}[r] \int_{\Omega_{\ell}} W_{2}[m] W_{2}^{T}[r] \mathrm{d} \Omega
\end{aligned}
$$


where the sum extends over all cells $\ell$ of the simulation domain, with $\left\{\Omega_{\ell}\right\}$ the volume of cell $\ell$ and $\hat{y}[m]$ the unit vector pointing in the direction of the edge associated to the Whitney form of first order $W_{1}[m]$ or of second order $W_{2}[m]$. The diagonal matrix $\phi$ is defined by

$$
\phi(\ell)=\left[\begin{array}{ccc}
\frac{s_{1}(\ell) s_{2}(\ell)}{s_{0}(\ell)} & 0 & 0 \\
0 & \frac{s_{2}(\ell) s_{0}(\ell)}{s_{1}(\ell)} & 0 \\
0 & 0 & \frac{s_{0}(\ell) s_{1}(\ell)}{s_{2}(\ell)}
\end{array}\right],
$$

which is the typical constitutive law of a uniaxial anisotropic medium representing the PML.

We can now apply the general subgridding scheme of Section 2.1 in a straightforward manner. By applying the same approach that lead to (4b) starting from $\left[\star_{\varepsilon_{r}}\right]$, the stretching matrices $\Lambda_{1}$ and $\Lambda_{2}$ in case of subgridding are transformed into

$$
\begin{aligned}
& \Lambda_{1}[j, k]=\sum_{r} \sum_{m} A_{1}[j, m] \tilde{\Lambda}_{1}[m, r] A_{1}^{T}[r, k] \\
& \Lambda_{2}[j, k]=\sum_{r} \sum_{m} A_{2}[j, m] \tilde{\Lambda}_{2}[m, r] A_{2}^{T}[r, k]
\end{aligned}
$$

If necessary mass lumping can be used to make the resulting matrices diagonal. In the PML regions, normal subgridding can now be embedded in the standard PML equations. From this point on, the classical discretization of the time derivative [?,?] is applied. This results, among others, in PML parameters taken exactly at the cell boundaries, thereby removing the need for approximate linear interpolations as in case of mass lumping. However, tangential subgridding excites spurious modes [?] within the PML. Since all PML theory assumes a source free PML medium, great caution should thus be taken when including tangential subgridding within a PML.

\section{NUMERICAL RESULTS}

We now carefully test the theory outlined in Section 2 First, we evaluate the generalized subgridding scheme of Section 2.1 for a closed simulation domain, terminated by PEC walls. Ideally, the subgridding should not cause any reflections at interfaces between meshes with different densities. A wave should travel from one submesh to another as if the refinement of the meshes did not matter. In practice, different refinement factors results in different grid densities and, hence, a different dispersion relation. Therefore, non-physical reflections may occur at the mesh interface. Second, we move to the open regions terminated by PMLs. We again study the reflection caused by the combination of the PML discretization and the subgridding of the PML region. Finally, the technique is put to the test in two realistic configurations: a differential stripline and differential microstrip interconnect.

\subsection{Spurious reflections introduced by the subgridding scheme}

Although the theory is formulated in $3 \mathrm{D}$, we will study reflection at a subgridding interface in a $2.5 \mathrm{D}$ (the $z$-dimension will be suppressed) configuration, in order to reduce calculation times and memory requirements. Two meshes $M^{1}$ and $M^{2}$ consisting of $330 \times 420$ and $90 \times 420$ coarse cells (Fig. 33), respectively, are stitched together, with the $x$-direction being the normal direction and the $y$-direction being the tangential direction. The coarse discretization length equals $\Delta x=\Delta y=\Delta=0.001 \mathrm{~mm}$ whereas $\Delta \tau(=c \Delta t)$ is chosen to be $288.675 \mu \mathrm{m}, 90 \%$ of the CFL-limit for a with $\nu=[2,2,2]$ subgridded mesh. The configuration is excited by a soft Gaussian line source (Gaussian pulse, GP, $z$-direction) in $M^{1}$ in the middle of the simulation domain, at coarse position $E_{z}^{[210,210]}$, with time variation

$$
G P(t)=A_{0} \nu_{x} \nu_{y} e^{-\frac{\left(t-t_{0}\right)^{2}}{\sigma^{2}}}
$$

with $A_{0}=0.25, t_{0}=200 \Delta t$ and $\sigma=66 \Delta t . \nu_{x}$ and $\nu_{y}$ are needed to scale the pulse's amplitude correctly in the fine mesh, as otherwise the amplitude of the soft source depends on the mesh 


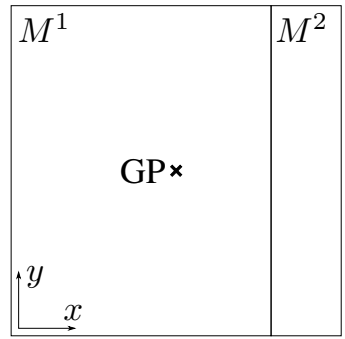

(a) 'cRef' set-up

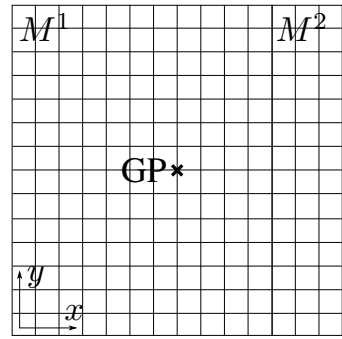

(e) 'fRef' set-up

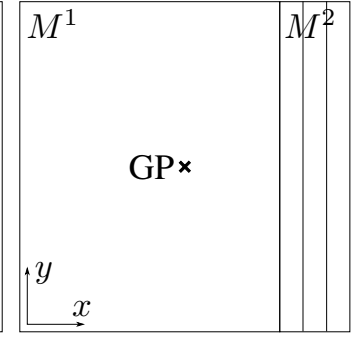

(b) 'cn' set-up

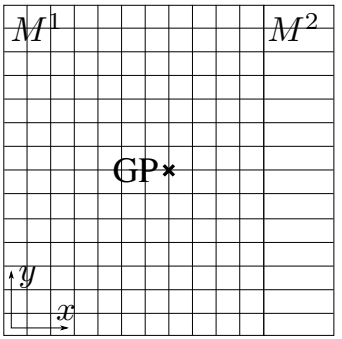

(f) 'fn' set-up

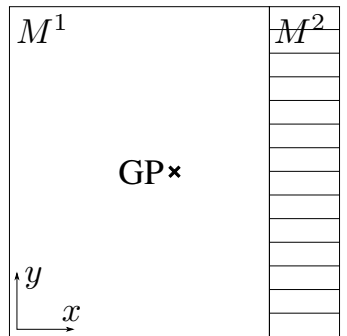

(c) 'ct' set-up

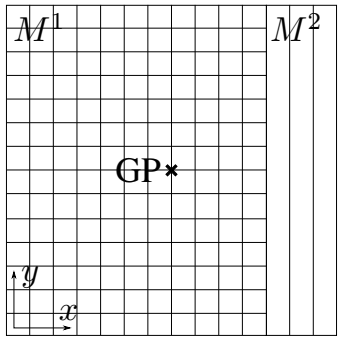

(g) 'ft' set-up

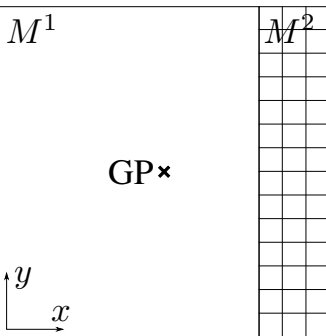

(d) 'cnt' set-up

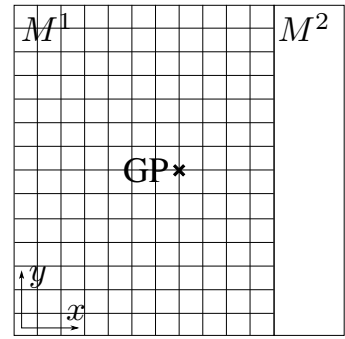

(h) 'fnt' set-up

Figure 3. Different subgridding schemes for an $M^{1}$ and $M^{2}$ mesh consisting of $330 \times 420$ and $90 \times 420$ coarse cells. $M^{1}$ has a fixed coarse (cases (a) to (d)) or fine (cases (e) to (h)) discretization. $M^{2}$ is (un)refined in either the normal (cases (b) and (f)), tangential (cases (c) and (g)) or both (cases (d) and (h)) directions.

discretization. The parameters are chosen in such a way that most of the frequency content of the GP lies below $10 \mathrm{GHz}$. At this upper frequency, the chosen discretization still provides 30 samples per wavelength. An observer is placed one coarse cell next to the source, co-located with the field $E_{z}^{[211,210]}$

Two sets of four different set-ups are considered (Fig. 3). For each set, a uniform reference configuration is defined. On the one hand, grid refinement (Fig. 3 $3 \mathrm{a}-\mathrm{d}$ ) is studied, starting from a fully coarse mesh 'cRef' (Fig. 3 3 ). On the other hand, grid unrefinement (Fig. 3e-h) is evaluated, using a fully fine mesh with $\nu=[2,2,1]$, 'fRef' (Fig. 3e), as a starting point. We investigate the reflections induced by refining or unrefining the mesh in the normal (Fig. 3.b) and Fig. 3(f)), tangential (Fig. 3.c) and Fig. 3. (g)) or both (Fig. 3.d) and Fig. 3.h)) directions.

3.1.1. Time-domain results First, a simulation over a very large number of time steps $100000 \Delta t$ is performed, while the energy as a function of time is stored, to test for instabilities. All tests resulted in constant energy, proving the stability of the proposed scheme. A formal proof can be derived, based on the theory outlined in [?].

Next, the results of subgridding are investigated in more detail. To do so, the solution of the corresponding coarse (Fig. 3a) or fine (Fig. 3e) reference configuration is subtracted from simulation results of the refined or unrefined configuration in the normal (cases (b) and (f)), tangential (cases (c) and (g)) or both (cases (d) and (h)) directions. Afterwards the logarithm is taken to emphasize the differences. By subtracting the reference solution, any reflection on the PEC-walls is removed. Around $800 \Delta t$ a first spurious signal, caused by the reflection at the subgridding interface between $M^{1}$ and $M^{2}$, is observed. One can clearly see in Fig. 4 that the tangential subgridding introduces a significantly smaller error than normal subgridding. The spurious signals around $1455 \Delta t\left(2^{\text {nd }}\right.$ reflection) are caused by the errors from the first subgridding interface crossing, followed by a reflection at the PEC-wall and then a second subgridding interface crossing. This explains why the second reflection is larger than the first. Note first that, overall, the errors are extremely small and second, the peak of the second reflection of the tangential subgridding is reached only $\approx 300 \Delta t$ after the peak of normal subgridding. 


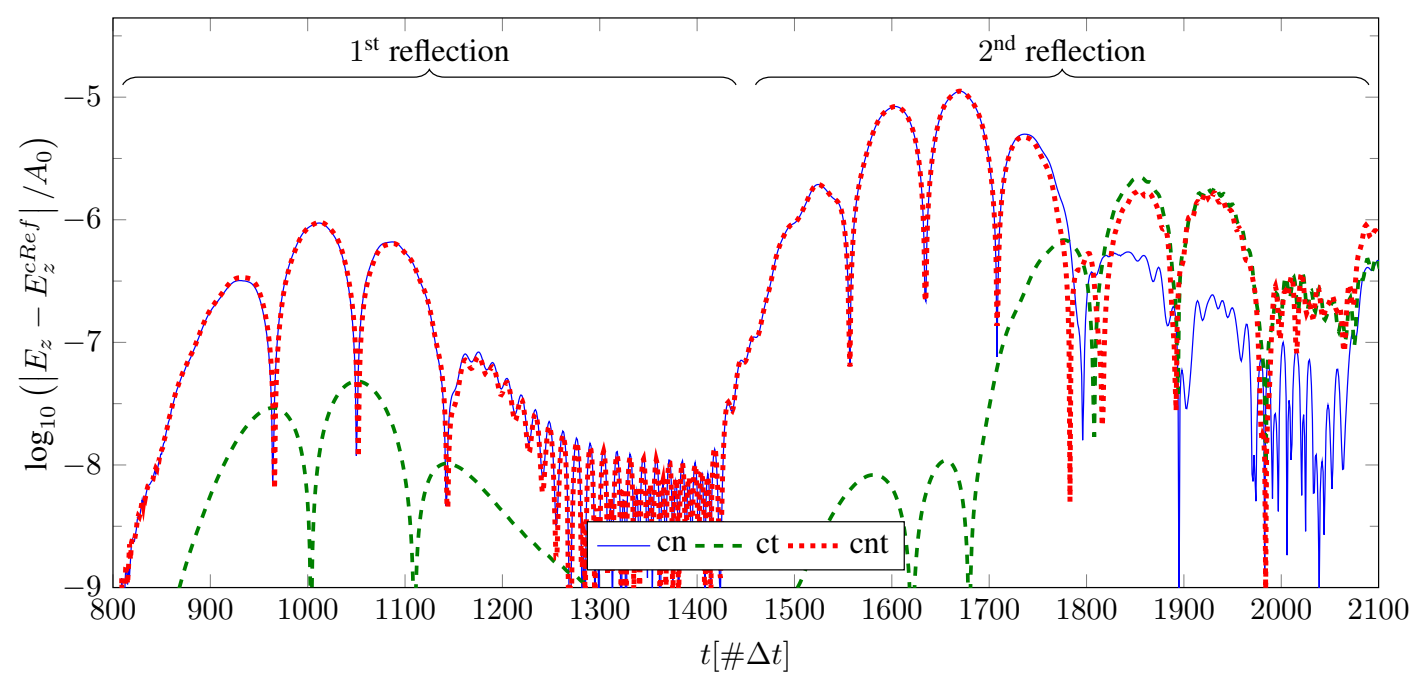

(a) wave travelling from coarse mesh $M^{1}\left(N^{1}=[1,1,1]\right)$ to refined mesh $M^{2}$

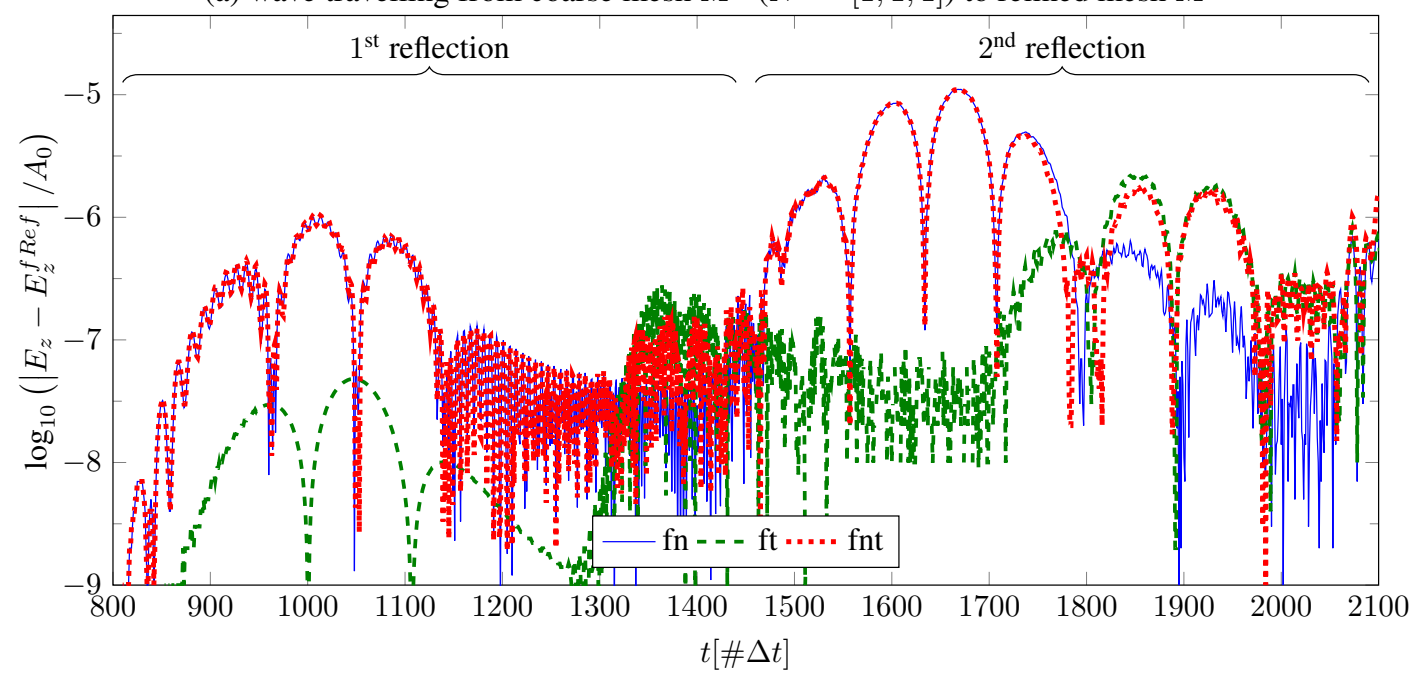

(b) wave travelling from a fine mesh $M^{1}\left(N^{1}=[2,2,1]\right)$ to unrefined mesh $M^{2}$

Figure 4. Errors normalized to $A_{0}$ (the amplitude of the Gaussian pulse) in the time domain due to reflections of a wave at the interface of a uniform mesh $\left(M^{1}\right)$ and a subgridded mesh $\left(M^{2}\right)$, (un)refined in the normal (label ' $\mathrm{n}$ '), tangential (label ' $\mathrm{t}$ ') or ('nt') both directions.

3.1.2. Frequency-domain results First, to convert the TD signal to the frequency-domain (FD), a 4096 point DFT is calculated, but only the first 1400 data points are used, to ensure the effect of only one subgridding interface crossing is taken into account. Next, a cut-off frequency $f_{c}$ is set at $15 \mathrm{GHz}$. Frequencies above $f_{c}$ are not of interest given the increasing grid dispersion error and, hence, will not be shown in graphs. Finally, he error is normalized to $A_{0}$, (the amplitude of the Gaussian pulse), to remove the influence of the input amplitude.

A quick look at the errors in the frequency domain confirms the time-domain result of Section 3.1.1. It is clear from Fig. 5 that tangential subgridding introduces significantly less errors than normal subgridding.

One can almost observe no difference between the absolute error induced by refining a coarse mesh (Fig. 5a) and the error induced by unrefining a fine mesh (Fig. 5b). Only around $10 \mathrm{GHz}$ the error induced by unrefining a fine mesh (Fig. 5b) in the tangential direction increases significantly 


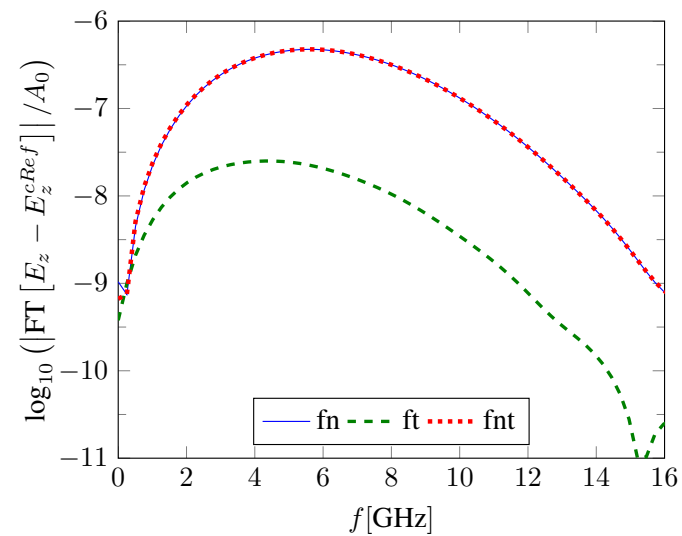

(a) Coarse reference mesh

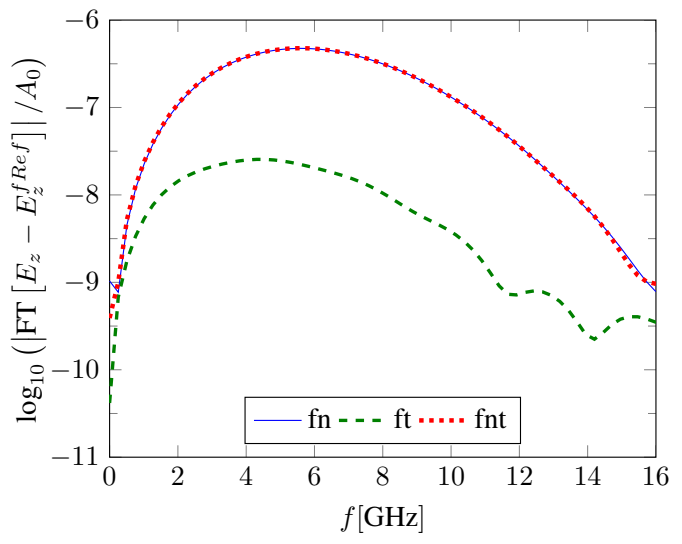

(b) Fine reference mesh

Figure 5. Error normalized to $A_{0}$ (the amplitude of the Gaussian Pulse) as a function of frequency when a wave travels from a uniform mesh $\left(M^{1}\right)$ to a subgridded mesh $\left(M^{2}\right)$, in case of (un)refining $M^{2}$ in the normal ('n'), tangential ('t') or both ('nt') directions.

compared to the error seen when refining a coarse mesh (Fig. 5a). This occurs because at higher frequencies the fine mesh suffers from less grid dispersion than the coarse mesh. In the coarse mesh, these higher frequencies experience a dispersion relation that differs from the free-space one and, therefore, additional errors occur. Note also that adding tangential subgridding to normal subgridding has almost no influence on the value of the error.

In general, we conclude that the subgridding algorithm is found to be stable and only introduces very small errors into the calculation domain. If the configuration under study allows for it, only using tangential subgridding results in smaller errors.

\subsection{Spurious reflections introduced by PMLs combined with subgridding}

Although not as perfect as predicted by the theory [?], in practice, PMLs give nowadays the best numerical approximation of free space for FDTD algorithms. Known advantages as low reflections, close application to scatterers, ... come with two major disadvantages, the calculation and storage cost. The PML update equation is computationally much more expensive than a standard update equation and a PML-field needs more storage than a standard field, next to the fact that extra layers of cells have to be added to the simulation domain. This means, on the one hand, that for small problems, relatively, a rather large part of the calculation time is spent within the PML. On the other hand, for large problems, the relative calculation effort decreases, but calculation time nevertheless increases noticeably as does the needed memory.

So far, PMLs have only been applied to uniform meshes. Therefore, the origin of reflections is the numerically imperfectly matched PML and, as a consequence, a part of the incident wave that is not totally absorbed. Applying a PML to a nonuniform mesh opens up some opportunities to reduce simulation time and memory consumption. First, one could add a uniform PML directly to the nonuniform mesh (Fig. 6a and Fig. 6b). This means that at points, the PML will be well matched to the mesh, but at other points additional mismatch will be introduced by the difference between the mesh and the PML discretization. Next, one could add a (few) uniform layer(s) of cells to the nonuniform mesh and then apply a PML. This means that reflections caused by subgridding and reflections caused by the PML are spatially separated, but this also means that additional storage and calculation efforts are needed. Finally one could apply a nonuniform PML (Fig. 6c), tailored to be less resource consuming.

We consider two configurations to investigate the termination by a PML when using a nonuniform mesh. Consider a 2D simulation geometry, discretized by a coarse mesh consisting of $420 \times 240$ cells in the middle and two fine meshes, both consisting of $420 \times 90$ coarse cells refined by a factor $\nu=[2,2,1]$, on top and below this centre region, as depicted in Fig. 7. The same Gaussian pulse 


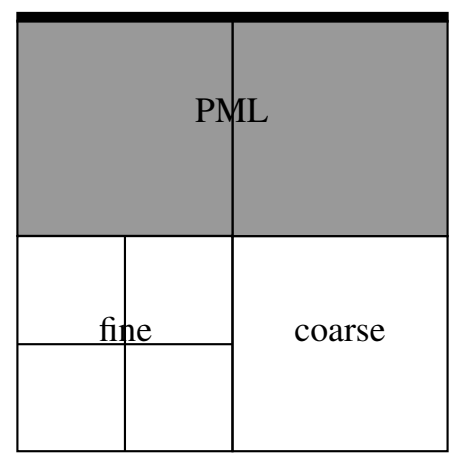

(a) Coarse PML

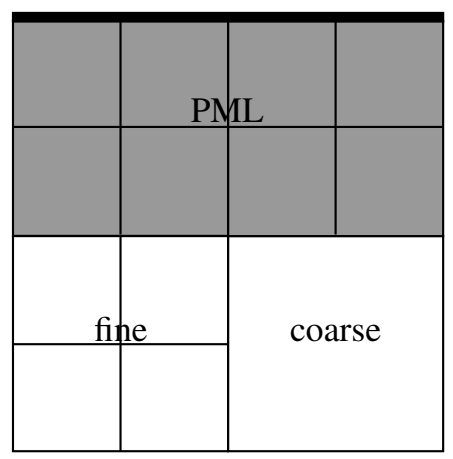

(b) Fine PML

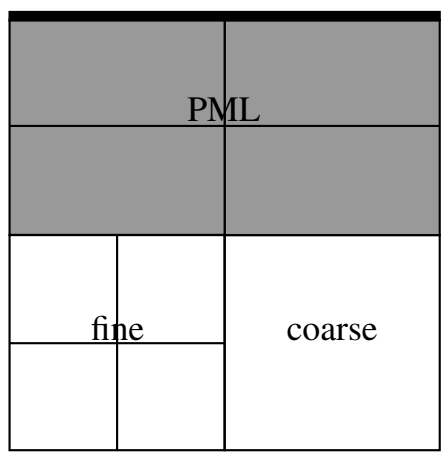

(c) PML that is unrefined in tangential direction

Figure 6. Schematic representation of different ways to terminate a nonuniform mesh (consisting of a fine and coarse submesh) at the top by applying a combination of refined and coarse PEC-backed PMLs (grey).

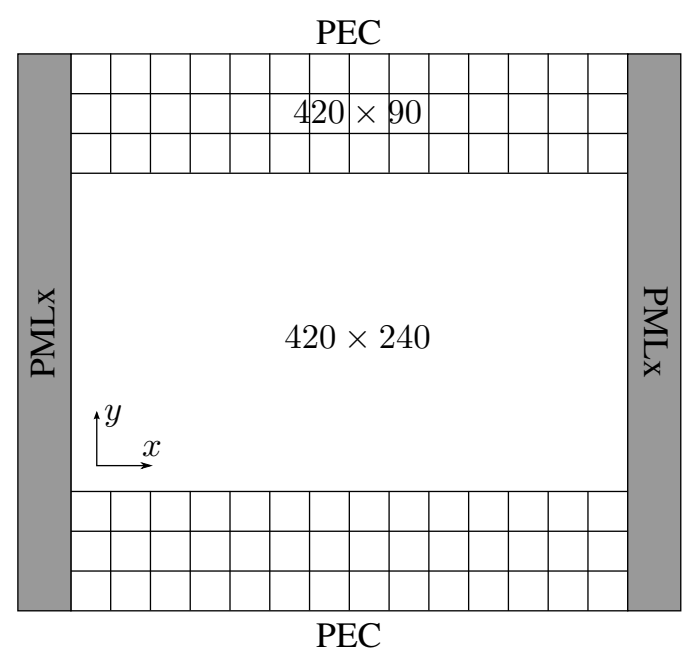

(a) mesh with PMLs in one direction

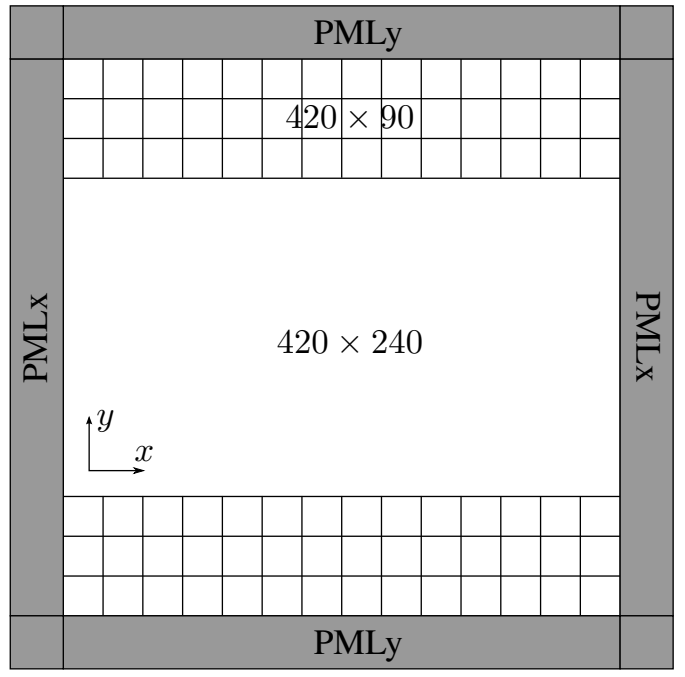

(b) mesh with PMLs in two directions

Figure 7. Subgridded mesh with overall size $420 \times 420$, consisting of a coarse inner mesh of size $420 \times 240$ and two outer meshes of size $420 \times 90$. All dimensions are expressed in coarse cells.

as in Section 3.1 is used to excite the configuration at its centre and again the observer is placed one coarse cell away from the source. We now create two kinds of open simulation domains by extending the configuration of Fig. 7 by means of PMLs. The first simulation domain is closed at the bottom and top by PEC plates and open at the side walls, by extending the mesh of these walls with a one-dimensional PML that only absorbs in the $x$-direction. For the second configuration, PMLs are added at all four walls, providing absorption in both the $x$ - and $y$-directions. To terminate the open regions, we apply a 5-layer PML, implemented as a UPML [?], with $\sigma$-profile

$$
\sigma_{o p t}=\frac{-(m+1) \ln R(0)}{2 Z_{c} \cdot 5 \Delta}
$$

where $R(0)=e^{-8}$ is the round-trip reflection for normal incidence and $Z_{c}$ is the impedance of free space. Each PML layer spans a single coarse mesh cell. In order to clearly isolate the effect of the subgridding, the same profile with $R(0)=e^{-8}$ is preserved also in case of a refined PML. The following types of subgridded PML are tested: a coarse-cell PML $(\nu=[1,1,1]$, labelled [111]), a fine-cell PML $(\nu=[2,2,1]$, labelled [221]) and a mixed-cell PML (labelled [dyn], having refinement 


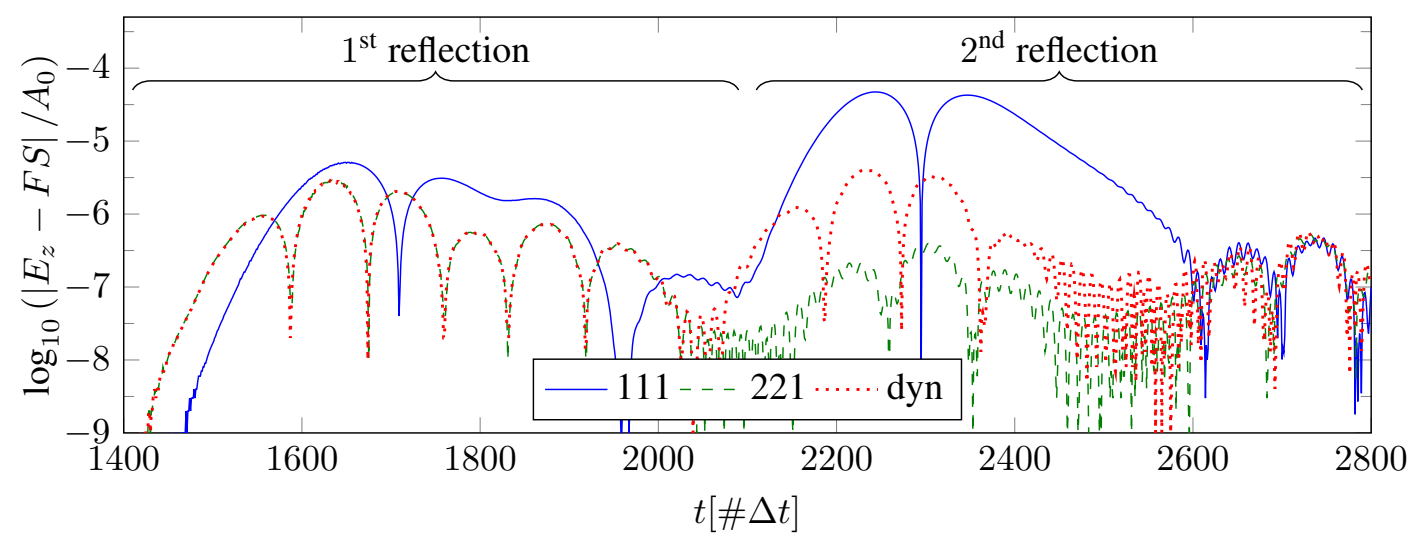

Figure 8. Errors normalized to $A_{0}$ (the amplitude of the Gaussian pulse) as a function of time in a slab-like mesh (Fig. 7a), terminated by differently discretized PMLs absorbing in one direction. The first reflection starts at $\pm 1450 \Delta t$, the second at $\pm 2100 \Delta t$.

factors $\nu=[2,1,1]$ when absorbing in the $x$-direction, $\nu=[1,2,1]$ when absorbing in the $y$-direction and $\nu=[2,2,1]$ when absorbing in both directions in corner regions). Note that the PML is unrefined in the tangential direction with respect to the fine mesh it interfaces with and that the discretization of the corner regions is refined in the normal direction with respect to the PMLs only absorbing in one single direction.

A reference solution is computed by enlarging the mesh in such a way that no reflections will enter the initial mesh for at least $3000 \Delta t$.

3.2.1. PML in one direction Consider Fig.77a, where the mesh is terminated by a coarse-cell ([111], Fig. 6a), fine-cell ([221], Fig. 6p) and mixed-cell ([dyn], Fig. 6r). The error signals with respect to the reference solution are plotted in Fig. 8. One can distinguish two main reflections. The first reflection occurs between $1450 \Delta t$ to $2000 \Delta t$, the time required for the wave to travel one round trip distance of $d \approx 420 \Delta$ between the source/observer and the PML. One notices that the finecell ([221]) and mixed-cell ([dyn]) PMLs exhibit the same level of error, clearly outperforming the coarse-cell ([111]) PML. The second reflection, taking place between $2100 \Delta t$ to $2700 \Delta t$ $(d \approx 595 \Delta \approx 420 \cdot \sqrt{2} \Delta)$ is caused by reflections at the corner points of the mesh, where the wave penetrated the PMLs under a much more oblique angle. For this reflection, the fine-cell ([221]) PML performs best, followed by the mixed-cell ([dyn]) and coarse-cell ([111]) PMLs. After the second reflection, all subgridded PMLs settle for the same error level.

3.2.2. PML in two directions In this section the mesh is terminated by the same PMLs but now in both $x$ - and $y$-direction (Fig. 7b). The errors generated by the different subgridded PML terminations with respect to the reference solution are shown in Fig. 9. The first reflection from both the $x$ - and $y$-directed PML reach the observer at the same time instant and will add up. The second reflection from the corners should, ideally, be absent, since it should be absorbed by the corner PML. Given the results obtained for a 1D PML, this is clearly expected for a fine-cell PML ([221]) and since all configurations in this section have fine-cell corner regions, we obtain very small second reflections for the PML configurations considered here. Fine-cell PMLs ([221]) outperform coarse-cell ones ([111]), as the latter additionally suffer from subgridding errors, but no relevant second reflection is observed. The mixed-cell PMLs ([dyn]) exhibit a similar performance as the fine-cell PMLs ([221]), yet they suffer from higher reflections from the corner regions. This is due to the fact that for the mixed-cell PMLs, the corner region is differently subgridded with respect to the $x$ - and $y$-directed PML.

We conclude that coarse-cell PMLs ([111]) use the least resources, but suffers from additional subgridding errors at the interface between the fine mesh and the PML. The fine-cell PMLs ([221]) perform best at the cost of a significant increase in computation time and memory resources. The 


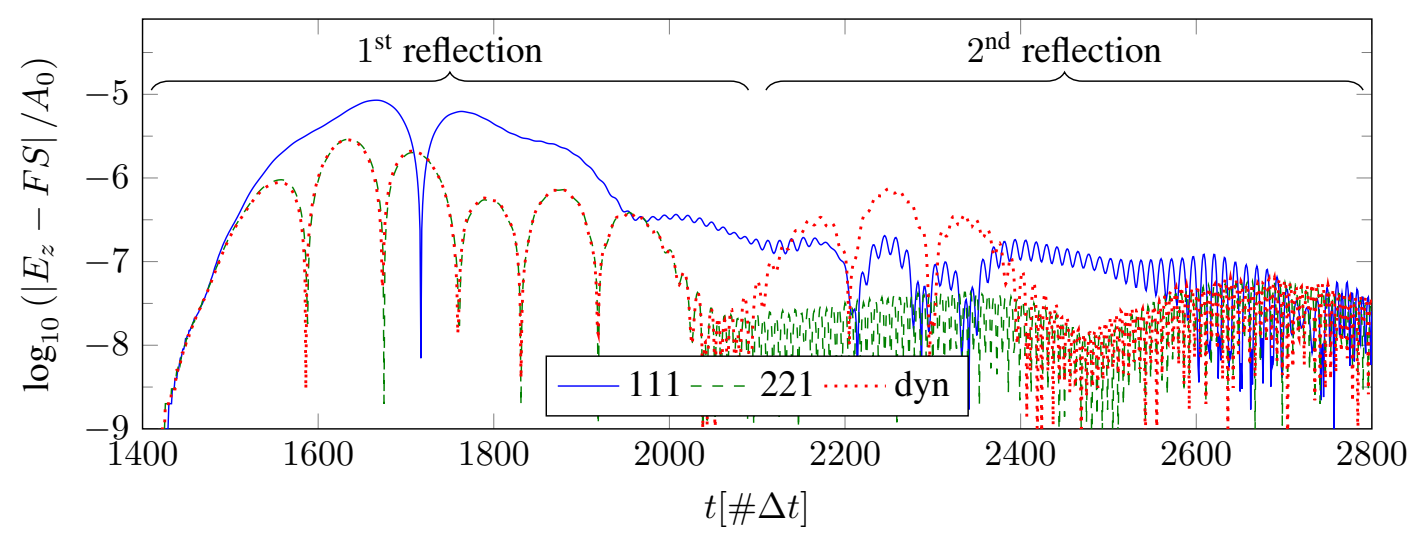

Figure 9. Errors normalized to $A_{0}$ (the amplitude of the Gaussian pulse) as a function of time in a slab-like mesh (Fig. 7b), terminated by differently discretized PMLs absorbing in two directions. The first reflection starts at $\pm 1450 \Delta t$, the second at $\pm 2100 \Delta t$.

mixed-cell PML ([dyn]) provides a good balance between accuracy and computational cost and is, therefore, the PML of our choice.

\subsection{Application example: differential stripline and microstrip interconnects}

Finally, we simulate an asymmetric differential stripline pair (Fig. 10a) and a differential microstrip (Fig. 10b) as realistic application examples that make use of both subgridding of the mesh and a PML as absorbing boundary condition. The interconnects were designed on a lossless Nelco ${ }^{\circledR}$ N4000-13 SI High-Speed Multifunctional Epoxy Laminate with relative permittivity $\varepsilon_{r}=3.2$. All conductors are assumed to be perfect electrically conducting.

First, we test the convergence of the solution in terms of the applied uniform discretization, by simulating the configurations once with discretization vector $\Delta_{1}=[15.1,507.8,9.0] \mu \mathrm{m}$ and once with $\Delta_{2}=[12.6,507.8,6.0] \mu \mathrm{m}$. These vectors were chosen such that the inner conductors of the stripline and the microstrip consist of an adequate number of cells, as further discussed, and because these discretizations yield an exact representation of the conductors with a minimal error on the spacing between conductors. For the remainder of this paper, results will be given with respect to $\Delta_{1}$, since the convergence experiments yielded consistent results for both discretizations. In the experiments, the differential pair is excited by a sinusoidal current with a frequency of $10 \mathrm{GHz}$. To reduce artefacts in the frequency domain, the sinusoidal excitation is ramped over five periods (or about $21000 \Delta t$, with respect to $\Delta_{1}$ ). During the simulations, both the current along and the voltage difference between the two conductors is measured over the full length of the stripline. The resulting time-domain vectors were then Fourier-transformed and subsequently matched to standing waves by means of the matrix pencil method [?]. By dividing the voltage by the current, the characteristic impedance of the stripline and the microstrip are determined. The obtained value will be compared to the impedance (later referred to as $Z_{r e f}$ ) as obtained by a dedicated Method of Moments technique [?, ?], developed to derive the modal parameters of high-frequency interconnects with arbitrary cross-sections.

3.3.1. Asymmetric differential stripline pair The configuration of the stripline with uniform discretization $\Delta_{1}$ yields conductors of size $10 \times y \times 2$ cells, spaced 12 cells apart. Now, a first reference solution is simulated without the use of PML absorbing boundary conditions, but by simply adding $1 \mathrm{~mm}$ (or 66 cells, after it was verified that increasing this distance has minimal influence on the simulation results) of substrate material to the left and the right of the inner conductors and terminating the complete simulation domain by PEC boundaries. This results in a calculation domain of $164 \times y \times 39$ cells. We now investigate the accuracy, by which the matrix pencil method is able to extract the modal parameters, as a function of the number of cells in the 


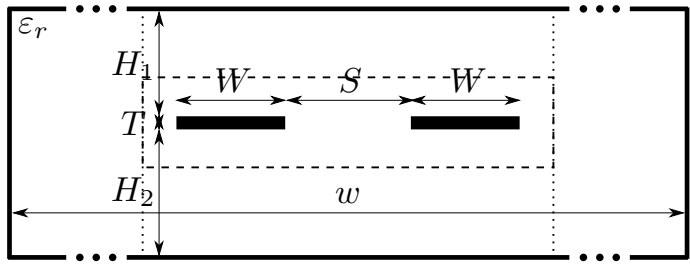

(a) Asymmetric differential stripline pair

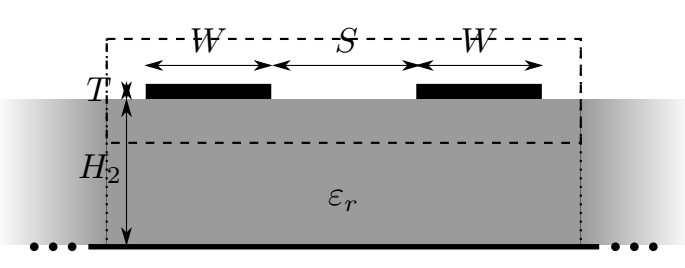

(b) differential microstrip

Figure 10. Asymmetric differential stripline and differential microstrip pairs with dimensions $H_{1}=150 \mu \mathrm{m}$, $H_{2}=180 \mu \mathrm{m}, T=18 \mu \mathrm{m}, W=151 \mu \mathrm{m}, S=175 \mu \mathrm{m}, w=2477 \mu \mathrm{m}$, in/on a Nelco ${ }^{\circledR}$ N4000-13 SI substrate with permittivity $\varepsilon_{r}=3.2$.

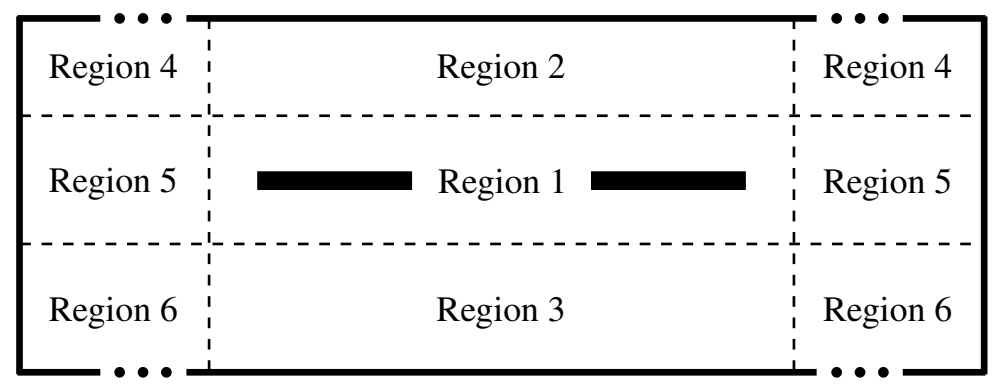

Figure 11. Subgridding regions to discretize the differential stripline pair

longitudinal direction $y$, trying to establish a good trade-off between accuracy and speed of the method. The initial settings for the reference simulation of the stripline corresponds to a length of three wavelengths (or 99 cells) and a duration of $100000 \Delta t$. The robustness of the matrix pencil method then allows reducing the stripline length to a length of only $\lambda / 4$ (or 8 cells). Further reducing the length below 8 cells, results in rank-deficient matrices in the matrix pencil method. A second set of experiments reduces the number of time steps. The results (with respect to $\Delta_{1}$ ) in Table [ s show that the best results are obtained for a simulation time of $50000 \Delta t$, while the worst results were obtained when simulating up to only $12500 \Delta t$. As expected, results deteriorate for shorter striplines simulated for shorter amounts of time. Remarkable is, though, that the results for a quarter wavelength stripline are distinctly better than, for example, a stripline of a third of a wavelength.Almost all simulations yield characteristic impedances that lie within $2 \Omega$ of the reference value.

Next we step-wise introduce subgridding and the subgridded PML boundary condition. First, we unrefine the standard (fine) reference solution (referred to as $Z_{\text {fine }}$ ), by limiting the fine mesh to a selected core region (within the dashed box in Fig. 10a and corresponding to 'region 1' in Fig. 11 with dimensions as indicated in Table (II), extending 2 and 3 coarse cells to the sides and to the top and bottom of the conductors, respectively, whereas the remainder of the calculation domain is unrefined with factors $\nu=[4,1,3]\left(Z_{s u b}\right)$, resulting in the mesh dimension in Table II In a second step, all cells left and right of the core region containing the conductors, being left and right of the dotted line in Fig. 10a, are replaced by a 5-cell PML. The stripline was simulated using both a coarse- $\left(Z_{[111]}\right)$ and mixed-cell $\left(Z_{[d y n]}\right)$ PML. The dimensions are again tabulated in Table II Table III contains the number of cells, field components and calculation time as well as the calculated impedances respectively. It can be seen that a distinct reduction in cells (and therefore field components) with a factor 5.8 has resulted in a time reduction with factor 4.8 . Table III] shows that all obtained impedances do not deviate much from $Z_{\text {ref }}$ and even less from $Z_{\text {fine }}$. 
3.3.2. Differential microstrip The configuration of the microstrip is identical to the one of the stripline with two obvious differences: the conductors are located on top of one Nelco ${ }^{\circledR} \mathrm{N} 4000$ 13 SI substrate with thickness $H_{2}=180 \mu \mathrm{m}$ and above the conductors there is an open free space region (or a PML in the simulation), meaning PMLs are now applied at the left, right and above the dotted box (see Fig. 10b). The distances kept from the conductors is 3 coarse cells at both the left and right side and 5 cells at the top. The coarse mesh, when applied, starts 2 coarse cells below the conductors. Table IV presents a comparison between the impedance obtained by our in-house Method of Moments technique, by the new subgridded FDTD techniques using a uniform fine mesh with matching fine-cell PML $\left(Z_{p m l}\right)$ and a nonuniform mesh with both coarse-cell and mixed-cell $\operatorname{PML}\left(Z_{[111]}\right.$ and $Z_{[d y n]}$, resp.). All simulations are obtained from a $\lambda / 3$-long microstrip simulated for $100000 \Delta t$.

The results presented above clearly demonstrate the stability of the proposed schemes. The obtained results hardly differ from a FDTD reference solution and are in good agreement with the results obtained from the in-house Method of Moments tool. Important here is that equally accurate results are obtained using 5.8 times less field components. This resulted in a significant speed-up (by a factor of 4.6) of the simulations.

\section{CONCLUSION}

In this paper, a framework for a stable, reciprocal and passive arbitrary subgridding procedure was presented. The consequences in terms of non physical reflections were studied and found to be of minor impact. In addition, a subgridding scheme for PMLs was proposed. It was found that an adequately subgridded PML provides a good balance between accuracy and calculation time. By means of two realistic examples, it was shown that the proposed strategies yield accurate results using significantly less resources both in terms of memory requirements and simulation time.

\section{ACKNOWLEDGEMENTS}

Funds for this research were obtained from the Agency for Innovation by Science and Technology in Flanders (IWT). 


\begin{tabular}{|c|c|c|c|c|c|c|}
\hline \multirow{2}{*}{\multicolumn{2}{|c|}{$Z_{\text {ref }}=49.21 \Omega$}} & \multicolumn{5}{|c|}{ length $[\lambda]$ (length [cells]) } \\
\hline & & $3.0(99)$ & $1.0(33)$ & $0.5(17)$ & $0.3(10)$ & $0.25(8)$ \\
\hline & $100 \mathrm{k}$ & 48.634 & 48.572 & 48.604 & 48.747 & 48.394 \\
\hline 1 & $50 \mathrm{k}$ & 49.217 & 49.266 & 49.209 & 49.266 & 48.817 \\
\hline \# & $25 \mathrm{k}$ & 47.947 & 47.035 & 47.216 & 45.693 & 50.130 \\
\hline E & $12.5 \mathrm{k}$ & 48.428 & 44.612 & 43.183 & 53.036 & 49.760 \\
\hline & $6.25 \mathrm{k}$ & 48.430 & 49.162 & 44.408 & 66.089 & 47.577 \\
\hline
\end{tabular}

Table I. Influence of the stripline length and the number of time steps on the calculation of the characteristic impedance $Z$.

\begin{tabular}{l|cccc} 
& fine & unrefined & PML [111] & PML [dyn] \\
\hline region 1 & $56 \times 15$ & $56 \times 15$ & $56 \times 15$ & $56 \times 15$ \\
region 2 & $56 \times 12$ & $14 \times 4$ & $14 \times 4$ & $14 \times 4$ \\
region 3 & $56 \times 15$ & $14 \times 5$ & $14 \times 5$ & $14 \times 5$ \\
region 4 & $52 \times 12$ & $13 \times 4$ & $5 \times 4$ & $20 \times 4$ \\
region 5 & $52 \times 15$ & $13 \times 5$ & $5 \times 5$ & $20 \times 5$ \\
region 6 & $52 \times 15$ & $13 \times 5$ & $5 \times 5$ & $20 \times 5$
\end{tabular}

Table II. actual mesh dimension (y-dimension suppressed), of the different simulated configurations

\begin{tabular}{|c|c|c|c|c|c|c|}
\hline & $Z_{\text {ref }}$ & $Z_{\text {fine }}$ & $Z_{\text {sub }}$ & $Z_{p m l}$ & $Z_{[111]}$ & $Z_{[d y n]}$ \\
\hline$\#$ cells $/ y$ & & 6396 & 1330 & 2772 & 1106 & 1526 \\
\hline gain $_{\text {cells }}$ & & 1.0 & 4.8 & 2.3 & 5.8 & 4.2 \\
\hline calc. time [s] & & 120341 & 28626 & 56092 & 25934 & 37734 \\
\hline gain $_{\text {time }}$ & & 1.0 & 4.2 & 2.1 & 4.8 & 3.2 \\
\hline$Z[\Omega]$ & 49.21 & 48.634 & 47.534 & 48.645 & 49.500 & 49.506 \\
\hline rel.err $Z_{\text {ref }}[\%]$ & 0 & 1.17 & 3.41 & 1.15 & 0.59 & 0.6 \\
\hline rel.err $Z_{\text {fine }}[\%]$ & 1.18 & 0 & 2.26 & 0.02 & 1.78 & 1.79 \\
\hline
\end{tabular}

Table III. Aggregated results for the asymmetric differential stripline

\begin{tabular}{|c|c|c|c|c|}
\hline & $Z_{\text {ref }}$ & $Z_{p m l}$ & $Z_{[111]}$ & $Z_{[d y n]}$ \\
\hline$Z[\Omega]$ & 67.85 & 68.399 & 67.644 & 67.328 \\
\hline rel.err $Z_{\text {ref }}[\%]$ & 0 & 0.81 & 0.3 & 0.77 \\
\hline rel.err $Z_{\text {fine }}[\%]$ & 0.8 & 0 & 1.1 & 1.57 \\
\hline
\end{tabular}

